\title{
Adhesion of Pollen Particles to Daily Disposable Soft Contact Lenses
}

This article was published in the following Dove Press journal: Clinical Optometry

\author{
Tatsuya Mimura' \\ Hiroshi Fujishima ${ }^{2}$ \\ Eiichi Uchio $\mathbb{D D}^{3}$ \\ Kazumi Fukagawa ${ }^{4,5}$ \\ Makoto Kawashima' \\ Kazuma Kitsu' \\ Hiroaki Horikawa' \\ Atsushi Mizota' \\ 'Department of Ophthalmology, Teikyo \\ University School of Medicine, Tokyo, \\ Japan; ${ }^{2}$ Department of Ophthalmology, \\ Tsurumi University School of Dental \\ Medicine, Yokohama, Kanagawa, Japan; \\ ${ }^{3}$ Department of Ophthalmology, Fukuoka \\ University School of Medicine, Fukuoka, \\ Japan; ${ }^{4}$ Ryogoku Eye Clinic, Tokyo, Japan; \\ ${ }^{5}$ Department of Ophthalmology, Keio \\ University School of Medicine, Tokyo, \\ Japan
}

Purpose: Pollen and proteins attached to soft contact lenses (SCLs) exacerbate allergic conjunctivitis. The material of SCLs may affect the pollen adhesion to the SCLs. The factors associated with the number of pollen particles that are adherent to daily disposable SCL were investigated.

Methods: Pollen particles were experimentally exposed to the contact lens surface of 12 types of SCLs for 1 hour, and the SCLs were washed and rinsed with a physiological saline $(n=10$ for each SCL type). A total of 120 contact lenses were used in this study. The pollen particles attached to the SCL were observed and photographed under a microscope. The influence of the materials of the SCLs on the degree of pollen adhesion were investigated.

Results: The number of residual pollen particles attached to SCLs was in the range from $0-293 /$ area of $200 \times 200 \mu \mathrm{m}$. Percentage of pollen adhesion area of the surface of the SCL was in the range from $0.01 \%$ to $3.25 \%$. There were significant differences in both the number and adhesion area of pollen particles among the 12 types of SCLs tested $(P<0.0001$ and $P<0.0001$, respectively). The number of pollen particles adhered to SCLs was significantly higher in colored SCLs than clear SCLs (unpaired $t$-test, $\mathrm{p}<0.001$ ). The portion of pollen adhesion area was the lowest in the silicone hydrogel SCLs made with delefilcon-A $(0.01 \pm$ $0.02 \%$ ).

Conclusion: Pollen adhesion in daily disposable SCLs depends on the characteristics and materials of the SCLs and was high in colored SCLs and lowest in delefilcon-A silicone hydrogel SCL. These results suggest that colored SCLs are not preferred during pollen season.

Keywords: daily soft contact lens, delefilcon-A, pollen, silicone hydrogel

\section{Introduction}

Seasonal allergic conjunctivitis, or hay fever, is the most common form of ocular allergy, affecting at least $15-20 \%$ of the population. ${ }^{1,2}$ Worn soft contact lenses (SCLs) accumulate tear components, ${ }^{3}$ including proteins, ${ }^{4,5}$ lipids, ${ }^{6}$ and mucins. ${ }^{7}$ Additionally, high water content SCLs (the Food and Drug Administration (FDA) Groups II and IV) generally accumulate greater amounts of deposits compared to low water content SCLs. ${ }^{5,6}$ The moist surfaces of SCLs may also trap various tear film components such as lipids, mucins and proteins. ${ }^{5}$ The proteins and lipids in tears attach to the SCL and may induce conjunctival inflammation. ${ }^{8-10}$ In addition, it can be hypothesized that allergic conjunctivitis may be exacerbated by foreign body reactions caused by just wearing the SCL or by pollens adherent to the SCL during hay fever season. Therefore, the SCLs on which pollen do not easily attach are better to maintain healthy eyes. Ueda and associates investigated the adhesion
Correspondence: Tatsuya Mimura

Tel +8I-3-3964-|2II

$\mathrm{Fax}+81-3-3964-1402$

Email mimurat-tky@umin.ac.jp
Clinical Optometry 2021:13 93-101 
rate of cedar pollen antigen Cry j1 to SCL. ${ }^{11}$ They reported that the SCLs that were replaced monthly had a significantly higher pollen adhesion rate than the daily exchange SCLs or the 2-week frequent replacement SCLs. ${ }^{11}$

It may be hypothesized that the materials of the SCLs can also affect the adhesion of pollen to the SCL. However, the effect of the material used for the daily disposable SCL on pollen adhesion has not been well determined. In Japan, SCLs made of delefilcon-A, etafilcon-A, narafilcon A, ocufilcon-D, ofhilafilcon-B, olifilcon $\mathrm{B}$, polymacon, samfilcon A, senofilcon-A, and stenfilcon A are mainly used. Polymacon belongs material group 1 of the FDA category, which is low water and non-ionic CL. Many hydrogel SCLs use polymacon (Table 1). SCL made of delefilcon-A material with unique water properties was recently introduced. Delefilcon-A is composed of a silicone-hydrogel core, with a $33 \%$ water content, and an outer hydrogel layer with an $80 \%$ water content. ${ }^{12}$

Thus, the purpose of this study was to determine the degree of pollen adhesion to disposable SCLs made of different materials.

\section{Methods}

\section{Research Design}

This was a non-clinical comparative effectiveness study. The procedures used in this study were approved by the Ethics Committee of Teikyo University. The title of this study was, "Study on the causative bacteria of ocular infections including contact lens contamination" (\#Teirin 18-227), and it was conducted at an ophthalmology laboratory of Teikyo University School of Medicine from April 2019 to April 2020.

OPTO_A_297531welve types of commercial daily disposable SCLs (all -4.0 diopters, each $n=10$ ) were used (Table 1). Therefore, a total of 120 contact lenses were used in this study. Japanese cedar pollen (Cryptomeria japonica) that were collected on March 3, 2019, were used (Yamizo Pollen Study Group, Daigo-cho, Ibaraki Prefecture, Japan). According to manufacturer instructions, pollen was shattered by drying male cedar flowers, and the pollen was naturally dried and purified with a filter (http://www.ne.jp/asahi/yamizo/kafun/).

\section{Adhesion of Pollen to SCLs}

One drop of $0.2 \mathrm{~mL}$ of physiological saline containing $0.01 \mathrm{mg} / \mathrm{mL}$ of cedar pollen was dropped on the contact lens surface of an unused SCLs $(n=10)$ and kept at room temperature for 1 hour as reported. ${ }^{14}$ After 1 hour, the SCLs were placed in a tube containing $10.0 \mathrm{~mL}$ of PBS and shaken for 10 seconds to remove pollens from the surface of SCLs. The SCL was further rinsed with a physiological saline three times. The measurement of the pollen particles was made after cleaning them 3 times with saline solution. The central part of the surface of the SCL was examined and photographed under a microscope. The number of particles adhering to an area of $200 \mu \mathrm{m} \times 200 \mu \mathrm{m}$ in the central part of the SCL and the portion of the adhering area were determined by ImageJ analysis software (version 1.52a; Wayne Rasband, $\mathrm{NIH}$, Bethesda, MD, http://imagej.nih.gov/ij/docs/index. $\underline{h t m l}$. It is impossible to take a picture of the entire SCLs with either a phase contrast microscope or an inverted microscope. Furthermore, in low magnification observation, the size of pollen particles became small and the image quality became rough, and then an analysis with Image J software was not possible. Therefore, only the image of the central part of SCLs could be analyzed. Figure 1 shows photographs of pollen particles after being left inside of SCL for 1 hour and after being rinsed with physiological saline.

\section{Statistical Analyses}

Two-tailed unpaired Student's $t$-tests were used to compare the mean values between two groups out of 12 groups. One-way analysis of variance and the KruskalWallis multiple comparison test were used to compare the mean values among three or more groups. The TukeyKramer method was also used to compare each group. Two lenses were selected from 12 types of contact lenses, and each two groups were compared in all combinations (66 ways). The correlations among variables were determined by calculating the two-tailed Pearson correlation coefficients and partial correlation coefficients. Factors associated with the number of pollen particles adhered to the SCLs were investigated by multivariate logistic regression analysis, with explanatory variables including water content (\%), oxygen permeability (Dk), oxygen transmissibility $(\mathrm{Dk} / \mathrm{L})$, diameter $(\mathrm{mm})$, base curve $(\mathrm{mm})$, colored SCL (clear $=0 /$ colored $=1$ ), and surface (ionic/non-ionic). The data are expressed as the means \pm standard deviations or percentages. Statistical analyses were performed with SAS System software version 9.1 (SAS Institute Inc., Cary, NC, USA), and significance was accepted at $P<0.05$. 


\begin{tabular}{|c|c|c|c|c|c|c|c|c|c|c|c|c|}
\hline 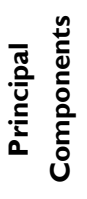 & 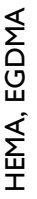 & 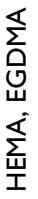 & 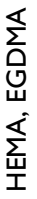 & 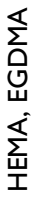 & 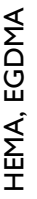 & 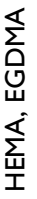 & 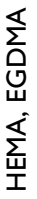 & 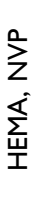 & $\begin{array}{l}\frac{\pi}{\Sigma} \\
\frac{\mathbb{L}}{\Psi}\end{array}$ & $\begin{array}{l}\frac{\pi}{\Sigma} \\
\frac{\mathbb{I}}{\Psi}\end{array}$ & 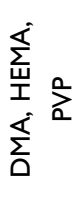 & 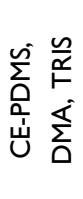 \\
\hline 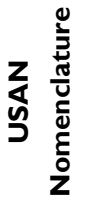 & 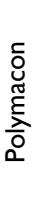 & 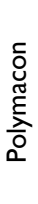 & 宅 & 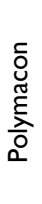 & 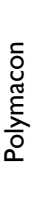 & 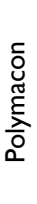 & 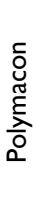 & 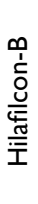 & 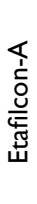 & $\begin{array}{l}0 \\
\frac{1}{0} \\
\frac{0}{E} \\
\frac{C}{3} \\
0\end{array}$ & 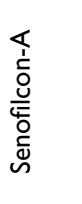 & $\begin{array}{l}\frac{1}{1} \\
\frac{1}{0} \\
\frac{0}{6} \\
\frac{0}{0} \\
\text { ด }\end{array}$ \\
\hline $\begin{array}{ll}\frac{1}{3} \\
1 & 0 \\
1\end{array}$ & - & - & - & - & - & - & - & $=$ & $\geq$ & $\geq$ & $>$ & $>$ \\
\hline
\end{tabular}

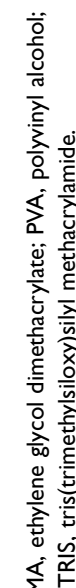

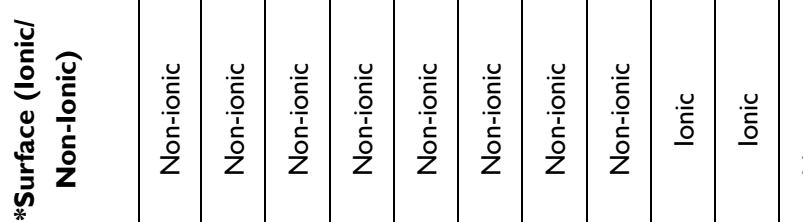

包蓠

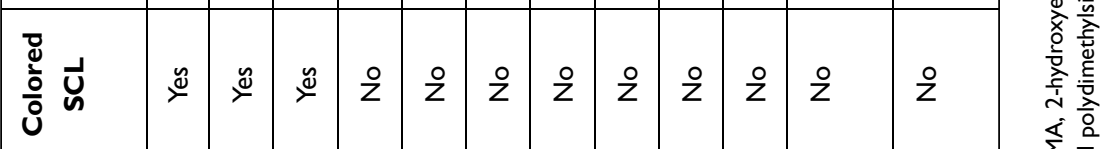

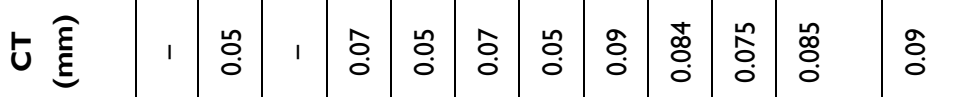

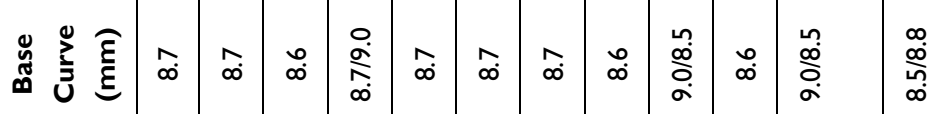

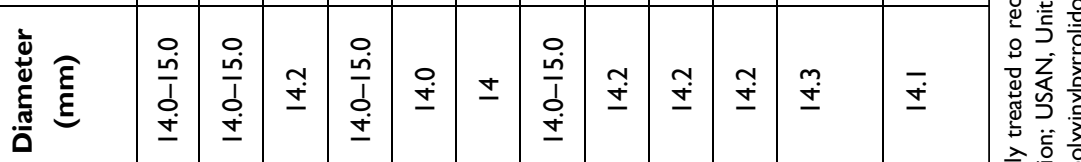

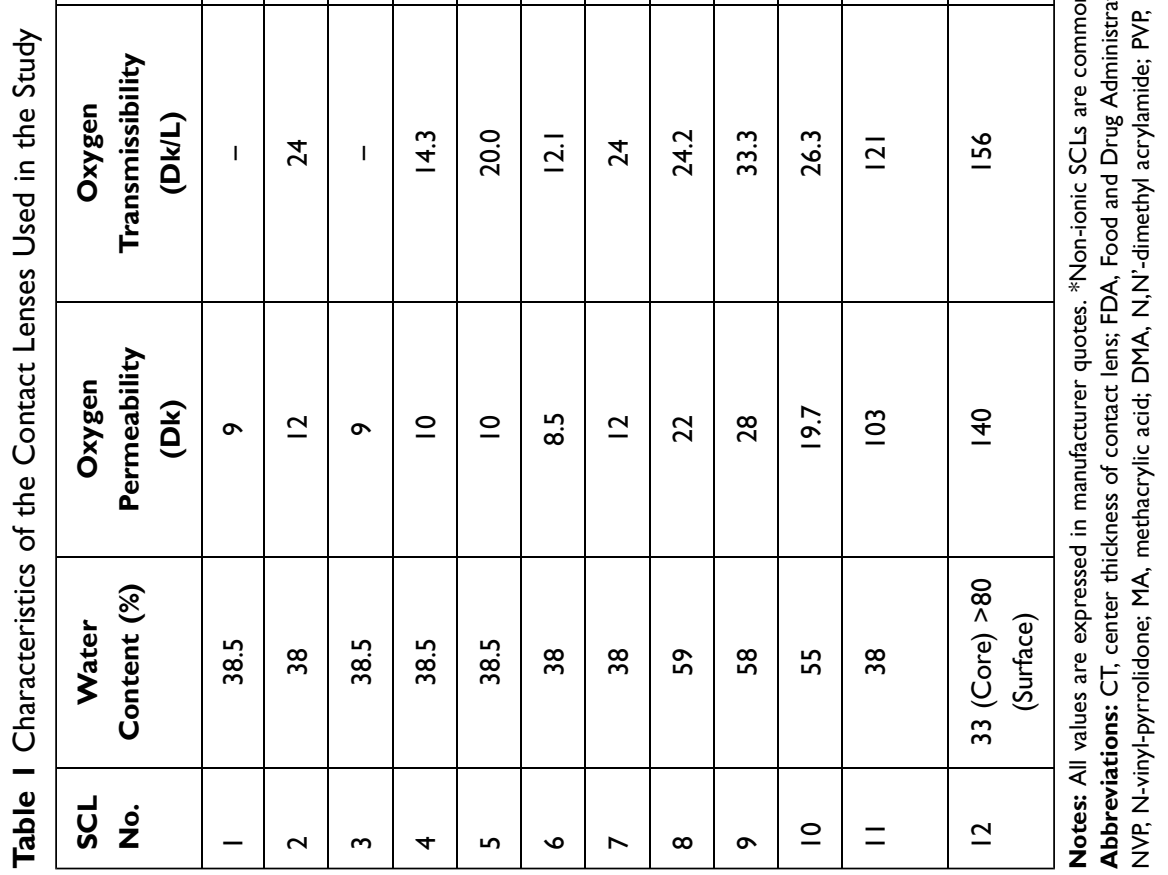




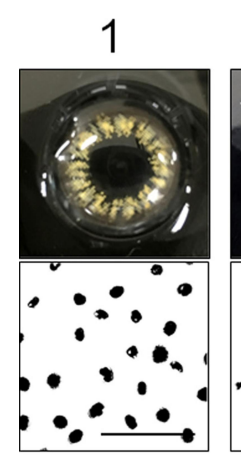

7
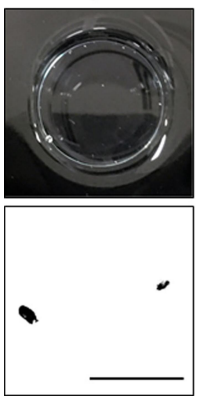

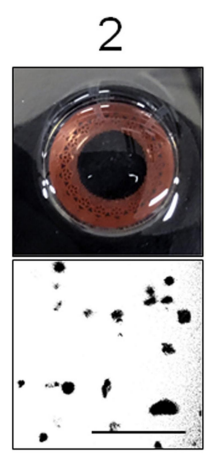

8
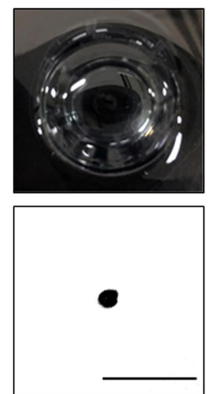

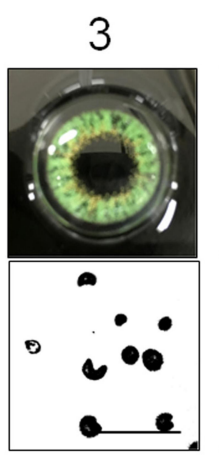

9

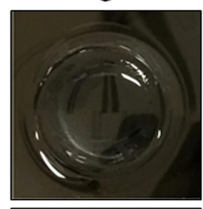

8

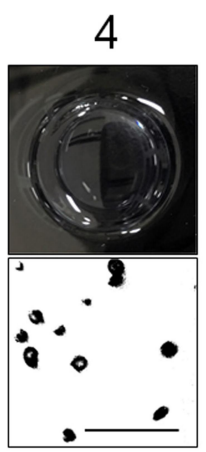

10
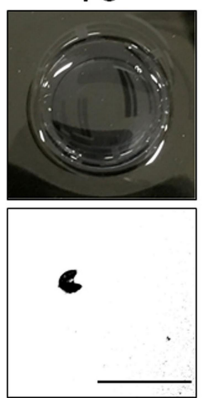

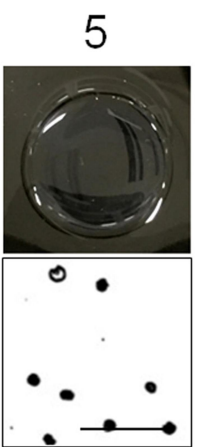

11
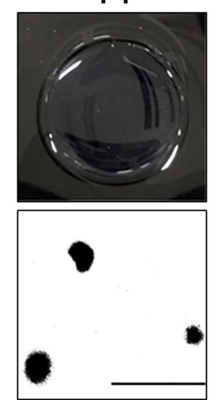

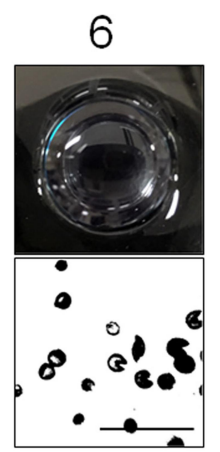

12
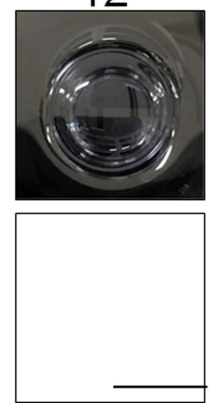

Figure I Photograph of pollen particles adhered to the surface of a SCL after one-hour exposure and extensive rinsing with physiological saline. Bars $=100 \mu \mathrm{m}$.

\section{Results}

\section{Numbers of Pollen Particles Adherent to} SCL

Photographs of the surface of SCLs and pollen particles adherent to the SCLs are shown in Figure 1. The number of pollen particles attached to a $200 \times 200$ um area on the SCLs are shown in Figure 2 and Table 2. There was a significant difference among the 12 groups $(P<0.0001)$.

\section{Degree of Pollen Particles Adherent to SCL}

The percentage of surface area that pollen takes up in a 200 x 200 um section of a SCL are shown in Figure 3 and Table 3 . There was a significant difference among the 12 groups (one-way analysis of variance, $P<0.0001$ ).

\section{Factors Affecting the Adhesion of Pollen Particles to SCLs}

The number of pollen particles that were adhered to the SCLs was negatively correlated with water content of the SCL ( $\mathrm{r}=-0.32, P=0.0003)$, oxygen permeability $(\mathrm{r}=$ $-0.25, P=0.0068)$, oxygen transmissibility $(\mathrm{r}=-0.22$, $P=0.0139)$, and diameter $(\mathrm{r}=-0.38, P<0.0001)$. The number was positively correlated with SCL color (circle

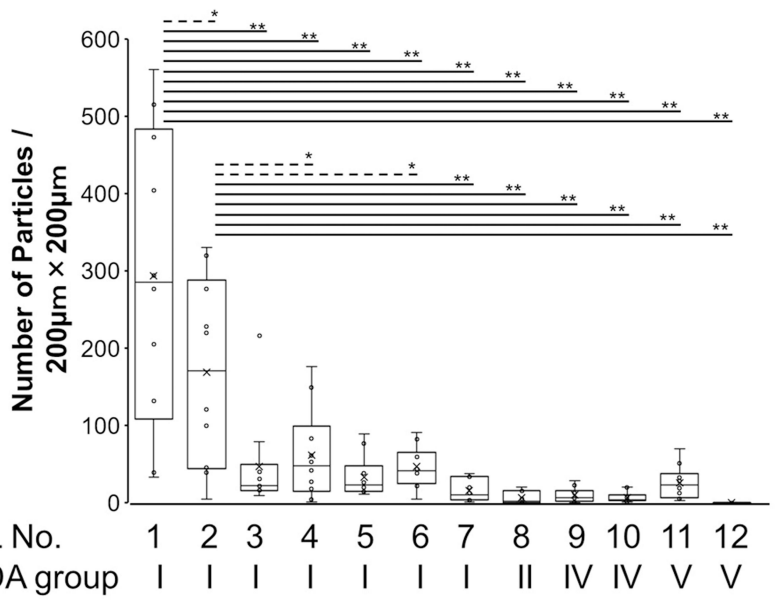

Figure 2 Number of pollen particles adhered to SCL/area of $200 \times 200 \mu \mathrm{m}$ in the central portion of the $\mathrm{SCL}$ after rinsing with physiological saline. The horizontal lines dividing the box indicate the median values. The vertical bars indicate the range and the horizontal boundaries of the boxes represent the first and third quartiles. The markers $(x)$ inside the box indicate the mean values. The mean values between two groups is compared by the Tukey-Kramer method $(* \mathrm{P}<0.05$ and $* * \mathrm{P}<0.01)$.

colored lens $>$ clear lens, $\mathrm{r}=0.59, P<0.0001$, two-tailed Pearson's correlation coefficient analysis, Table 4).

The correlations between the area of pollen adhered to SCLs and each characteristic of the SCLs are shown in Figure 4A-F. The portion of pollen adhesion area was significantly higher for colored SCLs (circle color lens, $2.46 \pm$ $2.19 \%)$ than in clear SCLs $(1.16 \pm 1.48 \%, P=0.0024$, 
Table 2 Number of Pollen Particles Attached to the SCL/Area of $200 \times 200 \mu \mathrm{m}$ Area

\begin{tabular}{|l|c|c|c|c|c|}
\hline SCL No. & Mean \pm SD & Range & Median & QI & Q3 \\
\hline 1 & $293 \pm 182$ & $33-560$ & 285.5 & 150.3 & 455.8 \\
\hline 2 & $169 \pm 115$ & $5-330$ & 170.5 & 59.5 & 264.8 \\
\hline 3 & $47 \pm 59$ & $9-216$ & 22.5 & 17.5 & 37.8 \\
\hline 4 & $61 \pm 56$ & $1-176$ & 47.5 & 20.3 & 77.5 \\
\hline 5 & $34 \pm 26$ & $11-89$ & 23.0 & 15.5 & 35.8 \\
\hline 6 & $47 \pm 25$ & $5-91$ & 41.5 & 29.0 & 59.8 \\
\hline 7 & $16 \pm 14$ & $1-38$ & 10.0 & 4.5 & 30.5 \\
\hline 8 & $6 \pm 8$ & $0-20$ & 2.0 & 0.0 & 12.5 \\
\hline 9 & $10 \pm 9$ & $0-29$ & 6.5 & 3.5 & 13.8 \\
\hline 10 & $7 \pm 7$ & $1-20$ & 3.5 & 3.0 & 6.8 \\
\hline 11 & $26 \pm 20$ & $3-70$ & 23.5 & 8.5 & 31.8 \\
\hline 12 & $0 \pm 1$ & $0-2$ & 0.0 & 0.0 & 0.0 \\
\hline
\end{tabular}

Abbreviations: SD, standard deviation; Q I and Q3, interquartile range Quartile I (QI) and Quartile 3 (Q3).

unpaired $t$-test). The portion of pollen adhesion area was significantly correlated with center thickness of SCLs, but not correlated with base curve of SCLs (Figure 4E and F).

The portion of pollen adhesion area was lower in the silicone hydrogel lens (SCL11 and SCL 12, FDA classification $\mathrm{V}, 0.33 \pm 0.54 \%$ ) compared with hydroxyethyl methacrylate-(HEMA-) based SCLs (SCL-1 - SCL-10,

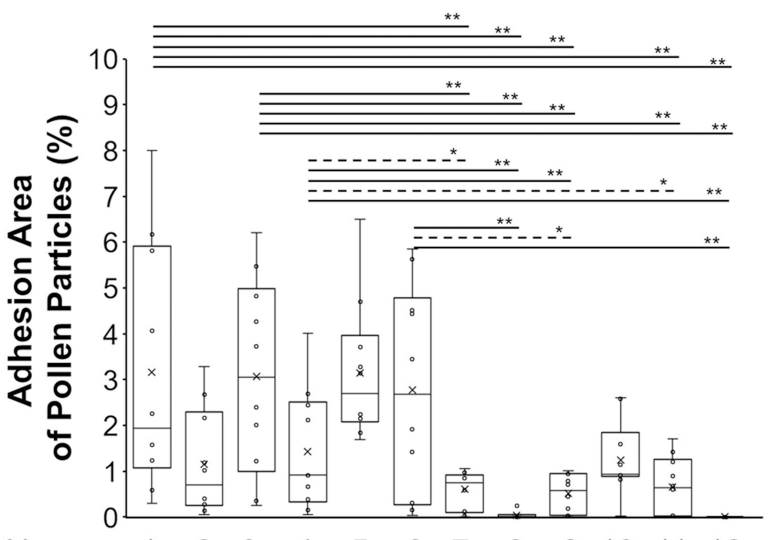

$\begin{array}{lllllllllllll}\text { CL No. } & 1 & 2 & 3 & 4 & 5 & 6 & 7 & 8 & 9 & 10 & 11 & 12\end{array}$ $\begin{array}{lllllllllllllll}\text { FDA group I } & \text { I } & \text { I } & \text { I } & \text { I } & \text { I } & \text { I } & \text { II } & \text { IV IV } & \text { V V } & \text { V }\end{array}$

Figure 3 The percentage of surface area that pollen takes up in a $200 \times 200$ um section of a SCL. The horizontal lines dividing the box indicate the median values. The vertical bars indicate the range and the horizontal boundaries of the boxes represent the first and third quartiles. The markers $(\mathrm{x})$ inside the box indicate the mean values. The mean values between two groups is compared by the TukeyKramer method $\left(* \mathrm{P}<0.05\right.$ and $\left.{ }^{*} * \mathrm{P}<0.01\right)$.
Table 3 Percentage of Adhesion Area of Pollen Particles on the Inner Surface of SCL (\%)

\begin{tabular}{|l|c|c|c|c|c|}
\hline SCL No. & Mean \pm SD & Range & Median & QI & Q3 \\
\hline 1 & $3.16 \pm 2.54$ & $0.30-8.00$ & 1.93 & 1.31 & 5.38 \\
\hline 2 & $1.15 \pm 1.11$ & $0.05-3.29$ & 0.71 & 0.29 & 1.92 \\
\hline 3 & $3.07 \pm 2.02$ & $0.26-6.21$ & 3.06 & 1.42 & 4.68 \\
\hline 4 & $1.44 \pm 1.24$ & $0.05-4.01$ & 0.92 & 0.45 & 2.37 \\
\hline 5 & $3.15 \pm 1.43$ & $1.69-6.50$ & 2.69 & 2.17 & 3.60 \\
\hline 6 & $2.77 \pm 2.16$ & $0.04-5.85$ & 2.68 & 0.59 & 4.49 \\
\hline 7 & $0.61 \pm 0.39$ & $0.01-1.05$ & 0.76 & 0.23 & 0.90 \\
\hline 8 & $0.04 \pm 0.07$ & $0.00-0.25$ & 0.01 & 0.00 & 0.05 \\
\hline 9 & $0.51 \pm 0.40$ & $0.00-1.01$ & 0.57 & 0.08 & 0.90 \\
\hline 10 & $1.24 \pm 0.77$ & $0.02-2.60$ & 0.93 & 0.91 & 1.48 \\
\hline 11 & $0.66 \pm 0.61$ & $0.00-1.71$ & 0.64 & 0.03 & 1.12 \\
\hline 12 & $0.01 \pm 0.02$ & $0.00-0.05$ & 0.00 & 0.00 & 0.00 \\
\hline
\end{tabular}

Abbreviations: SD, standard deviation; QI and Q3, interquartile range Quartile (QI) and Quartile 3 (Q3).

$1.71 \pm 1.85 \%, \mathrm{p}=0.0013)$. The portion of pollen adhesion area was lowest for the silicone hydrogel SCLs made with delefilcon-A $(0.01 \pm 0.02 \%)$.

\section{Discussion}

This is the first study that verifies the degree of pollen adhesion to 12 different 1-day disposable SCLs. The number of pollen particles varied considerably from 0 to 293 particles per $200 \times 200$ um area on the SCLs, but was higher in colored SCLs, and was lower in the silicone hydrogel SCLs that are classified as FDA V. The highest number of pollen was found on Polymacon SCLs while the fewest number was found on delefilcon-A SCLs.

The pollen particles remained attached to the surface of SCLs after rinsing 3 times but most of the pollen were removed by rubbing the SCL strongly with the fingers (Figure 5). Ueda et al investigated the adhesion of pollen to SCLs by immersing the SCLs in physiological saline containing pollen for 10 minutes and rinsing. ${ }^{11}$ They reported that pollen particles were almost completely removed by rinsing. Ueda's study and current findings indicate that the pollen may have poor adhesion to SCLs. In most plant species, the pollen is primarily highly viscous and sticky pectin with cellulose and hemicellulose. ${ }^{13}$ After the pollen bursts (hatch out), its adhesion becomes stronger. ${ }^{13}$ In this experiment, most of the pollen did not 
Table 4 Relationship Between the Number of Pollen Particles Adhered to SCLs and SCL Parameters, as Well as the Results of Multivariate Analysis

\begin{tabular}{|l|l|l|l|l|c|}
\hline \multirow{2}{*}{ Variables } & \multicolumn{3}{|c|}{ Correlation Coefficients } & \multicolumn{2}{c|}{ Multivariate Analysis } \\
\cline { 2 - 6 } & $\mathbf{R}$ & $\mathbf{( 9 5 \% ~ C l )}$ & P value & OR & P value \\
\hline Water Content (\%) & -0.32 & $(-0.48-0.15)$ & 0.0003 & 7.0 & 0.2268 \\
\hline Oxygen Permeability (Dk) & -0.25 & $(-0.41-0.07)$ & 0.0068 & 1.2 & 0.9653 \\
\hline Oxygen Transmissibility (Dk/L) & -0.22 & $(-0.39-0.05)$ & 0.0139 & 0.0 & 0.1328 \\
\hline Diameter (mm) & -0.38 & $(-0.52-0.21)$ & $<0.0001$ & 0.0 & $<0.0001$ \\
\hline Base Curve (mm) & -0.01 & $(-0.19-0.17)$ & 0.9375 & $>100.0$ & 0.3182 \\
\hline
\end{tabular}

Notes: Correlations between the number of pollen particles adhered to SCLs and parameters of SCLs were calculated with the two-tailed Pearson's product moment formula. Independent determinants of the number of adhered pollen particles were investigated by multiple logistic regression analysis. $\mathrm{R}=$ Pearson's correlation coefficient. Abbreviations: $\mathrm{Cl}$, confidence interval; OR, odds ratio.

burst (Figure 1), suggesting that adhesive property of the pollen to SCLs may have been reduced. Luensmann and associated reviewed and summarized the impact of SCL material compositions on the adsorption behavior of the protein albumin. ${ }^{5}$ The level of protein deposition on SCLs is strongly influenced by the tear composition and the chemical characteristics of the SCLs material such as, water content, pore size, roughness of the surface, hydrophobicity, and electrostatic charge, which are all important factors to attract protein on the surface of SCLs. ${ }^{5}$ The unburst pollen has poor adhesion to SCLs. However, as mentioned above, pectin localized in the inner intine layers of burst pollen may be involved in adhesion to SCLs. The moist surfaces of SCLs may trap various allergens, such as pollens, as well as protein. Therefore, the material composition of the SCLs such as the water content, oxygen permeability, and oxygen transmissibility may affect the attraction behavior of pollen, much like previous studies have demonstrated with albumin and SCL surface properties. $^{5}$
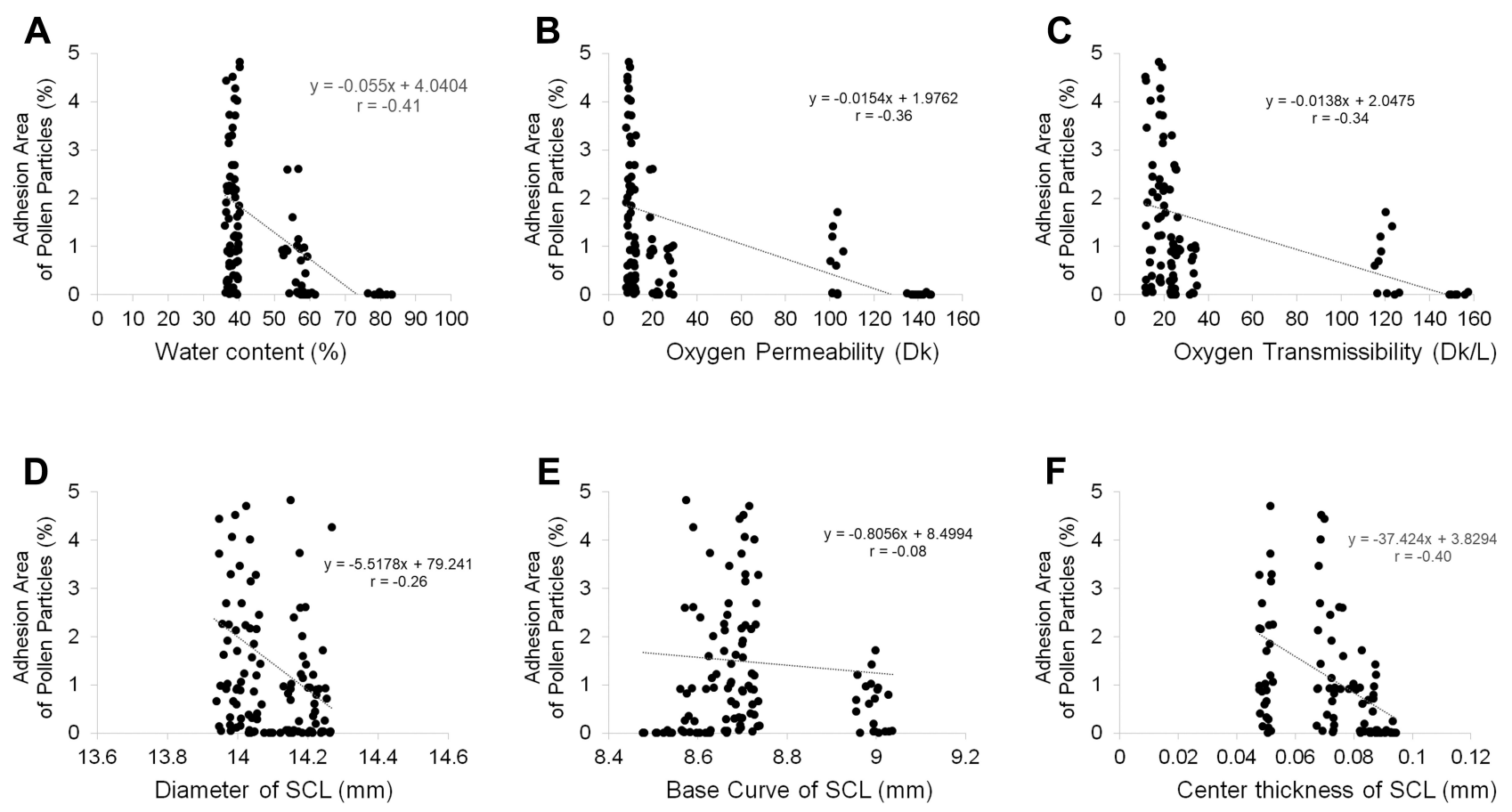

Figure 4 Relationship between the adhesion area of pollen particles to the SCLs and various parameters of SCLs. The plots are displayed as jitter plots arranged randomly, which avoids overlap of identical values. (A) Water content (\%). (B) Oxygen permeability (Dk). (C) Oxygen transmissibility (Dk/L). (D) Diameter of SCLs (mm). (E) Base curve $(\mathrm{mm})$. (F) Center thickness of SCLs $(\mathrm{mm})$. 

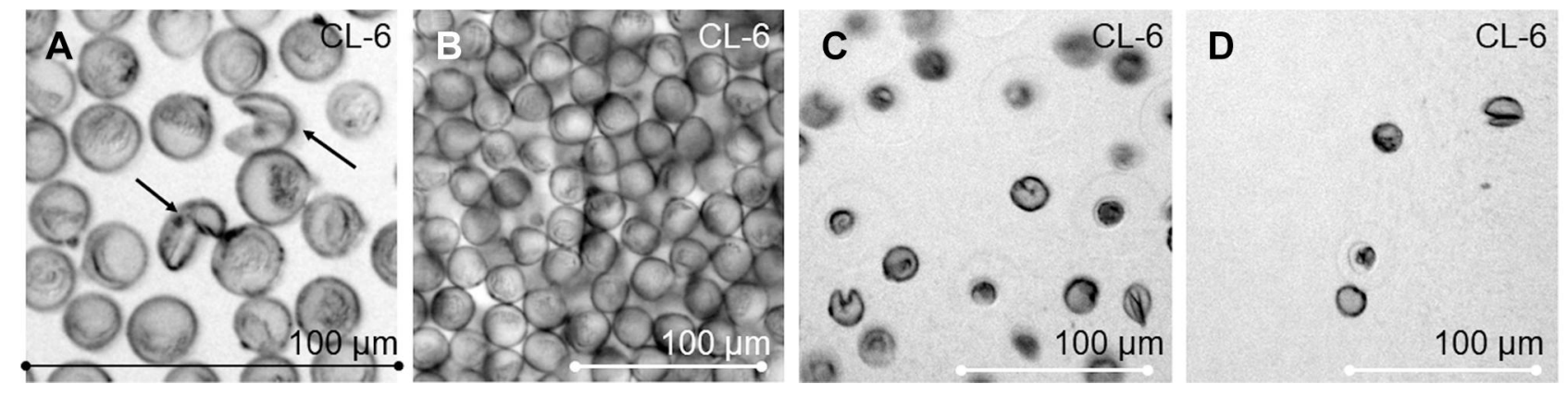

Figure 5 Photographs of pollens adhered to the inner surface of soft contact lenses (SCLs) (No. 6) (A) Photograph of burst (arrows) and unburst pollens on an SCL. (B) Pollen on an SCL for before washing. (C) Pollen on the SCL after rinsing without rubbing. (D) Pollen are removed by rubbing with the fingers. Bars $=100 \mu m$.

The physical properties of the SCLs such as the water content, oxygen permeability, and oxygen transmissibility were examined. Pollen adhesion was higher in colored SCLs and low water content SCLs classified in FDA in Group 1 (Figure 4A). The water content and the oxygen transmissibility are generally correlated with the water properties of the SCL materials in the 2-hydroxyethyl methacrylate (HEMA)-based SCLs. ${ }^{14}$ The reason why pollen adhered more strongly to FDA Group 1 SCLs may be related to their non-ionic surface treatment and their low water content.

The surface properties of SCLs such as hydrophilicity, roughness/smoothness, and coating may also affect the degree of pollen adhesion. The hatched pollen has higher adhesion to SCL but if there is much pollen that has not been hatched as in the cases, the adhesion rate may have been lower. Hydrogel SCLs are classified as either nonionic (FDA Groups I and II) or ionic (FDA Groups III and IV). Non-ionic hydrogel SCLs are treated to reduce the negative surface charge, therefore they are less likely to attract protein deposits. ${ }^{5}$ On the other hand, ionic lenses are charged with negative ions, thus will attract positively charged proteins in the tear film. Most pollen is charged and has either positive or negative electrostatic charge. ${ }^{15,16}$ This means that ionic SCLs with negative ions may attract positively charged pollen. Contact lens material affects roughness and smoothness of SCL surface. SCL is made by three different manufacturing processes (lathing, spin casting, and cast molding), leading to different roughness and smoothness values for the SCLs. ${ }^{17,18}$ SCLs manufactured by lathe-cutting exhibit notable higher roughness values of lens surfaces than those manufactured by spin casting or cast molding. ${ }^{17,18}$ Regarding the type of lens, Filcon $1 \mathrm{~A}$ and Filcon $4 \mathrm{~A}$ manufactured by the lathing method are reported to have the roughest surface. ${ }^{18}$ In cast molded SCLs, surface roughness decreased with increasing water content. ${ }^{18}$ Surface roughness of SCLs may enhance pollen adhesion. However, most modern SCLs are cast-molded. Therefore, the surface of any SCL is smooth and there may be no difference in smoothness between SCLs. The information on the surface treatment and manufacturing processes of the SCLs used in this experiment were searched, but details of the surface treatment were not reported in the instruction manual of the SCLs. Therefore, variables relating to SCL surface treatment was not included in the explanatory variables in the statistical analyses.

Three types of color SCLs were included in the group of non-ionic SCLs (No.1-3, Table 2). One type of color SCL has ink printed on the surface, which may attract proteins. The other two types of SCLs are manufactured through sandwich method to prevent the color in contact with eyes. The details of these three SCLs have not been revealed by the manufacturer. It may be speculated that the adhesion of proteins and pollen may be affected by the deposition of color ink on the SCL surface and the ink coloring method in the manufacturing process of SCLs.

The SCLs used in this study were very popular SCLs available in Japan and do not encompass the SCLs for all FDA types. Conventional SCLs are made mainly from a hydrogel material called HEMA. HEMA is a high-water content material that becomes soft when it contains water. ${ }^{19}$ Oxygen passes through the SCLs through the water contained in HEMA and reaches the cornea. ${ }^{19}$ Thus, in general, the higher the water content in a HEMA SCLs, the more oxygen reaches the cornea. ${ }^{19}$ On the other hand, the silicone hydrogel SCLs provide extremely high oxygen permeability value even if they have a low water content. ${ }^{20}$ Silicone hydrogel is a material that can overcome the problems of HEMA, but the hardness unique to silicone may be 
a disadvantage. ${ }^{21}$ Generally, silicone hydrogel SCLs have less protein adhesion than SCLs made with HEMA material. $^{22}$

Furthermore, among the silicone hydrogel lenses, the delefilcon-A SCLs (CL-12) have predominantly fewer pollen particles than with senofilcon-A (CL-11; Figures 2 and $3)$. The characteristic of this SCL constructed with delefilcon-A is that the front and back surfaces are composed of a hydrogel layer (water content of $80 \%$ or more), while the inner lens core is composed of hydrated silicone hydrogel (water content of 33\%). In other words, this lens is made with by a new technology called "three-layer moisture hydrogel". 23,24 The silicone materials are naturally hydrophobic. Therefore, the internal silicone hydrogel is coated with a hydrogel-rich water to improve the wettability of the surface of the lens. The SCLs are also coated with various substances after immersion in a dispersion of positively charged particles $\left(\mathrm{DOWEX}^{\mathrm{TM}}\right){ }^{23}$ Therefore, positively charged particles such as pollen do not easily adhere to this type of lens.

This study has many limitations. First, this study verified pollen adhesion to SCL only 1 hour after pollen instillation. At 1 hour, less pollen had burst and it may be necessary to confirm pollen adhesion to SCLs using burst pollen. Second, pollens were experimentally attached to SCLs which is far from the clinical situations. The amount of pollen scattered in Tokyo in March 2019 was about 100 particles $/ \mathrm{cm}^{2}$ per day. While, in this experiment, approximately 2500 pollen $/ \mathrm{mm}^{2}$ were dropped on the surface of SCLs. Clinically, so much pollen cannot adhere to the surface of the eye. Various protein and lipid components originating from the conjunctiva may be present in the tear fluid when the patients wear SCLs. Studies using pollen mixed with human tears are closer to actual clinical practice. However, in this study, a saline solution containing pollen was used instead of tear fluid. According to a report by Ueda et al, a maximum of 50 pg of Cry $\mathrm{j} 1$ per lens was attached to the SCLs with tear proteins and lipids worn by the subjects during the cedar pollen scattering season. Proteins and lipids in tear fluid attached to contact lenses may increase pollen adhesion to SCLs. ${ }^{11}$ Third, the study used only daily disposable SCL. The daily disposable SCLs need only be discarded, but 2-week and monthly frequent replacement SCLs or conventional SCLs require daily cleaning of proteins such as pollens. Therefore, rather than a daily disposable SCLs, a study using the twoweek type or conventional SCLs may be useful. Forth, this study cannot discuss how pollen sticks to the eye. So far, no research has been done on the adhesion of pollen to the eye. Fifth, in this experiment, we could not examine the mean number of pollens in the peripheral area of the contact lens because the periphery of the contact lens was curved. Since the surface of the contact lens makes oblique contact with the objective lens of the microscope, it was theoretically impossible to observe the peripheral part of the contact lens under the microscope. Sixth, we could only observe pollen on contact lenses with a $4 x$ objective lens. It was difficult to take a picture even with a $4 x$ lens, because the contact lens is curved, and the peripheral part of the contact lens adhered to the objective lens. The pollen and contact lenses were completely adhered to the surface of the 10x objective lens and could not be photographed. The pollen size was about $20 \mu \mathrm{m}$ and the contact lens thickness was 50-90 $\mu \mathrm{m}$. The working distance of the 10x objective lens of our microscope was only $45 \mathrm{~mm}$. Therefore, it was impossible to observe large pollen attached to the contact lens surface under the microscope with a 10x objective lens.

In recent years, silicone hydrogel SCLs have been greatly improved to suppress the onset of giant papillary conjunctivitis. In this study, pollen particle adhesion was extremely low in the silicone hydrogel SCL with delefilcon-A. The conclusion of this study is that SCLs with clear color, thick center thickness, non-ionic, high water content, and high oxygen transmissibility are less prone to pollen adhesion.

\section{Funding}

This work was supported in part by a Grant-in-Aid for Scientific Research from the Ministry of Education, Culture, Sports, Science and Technology of Japan [grant number 20H04347] and an unrestricted investigatorinitiated grant from Alcon Japan Ltd. to Tatsuya Mimura, MD. The funders had no role in study design, data collection and analysis, decision to publish, or preparation of the manuscript.

\section{Disclosure}

The authors report no conflicts of interest in this work.

\section{References}

1. La Rosa M, Lionetti E, Reibaldi M, et al. Allergic conjunctivitis: a comprehensive review of the literature. Ital J Pediatr. 2013;39:18. doi:10.1186/1824-7288-39-18

2. Wong AH, Barg SS, Leung AK. Seasonal and perennial conjunctivitis. Recent Pat Inflamm Allergy Drug Discov. 2009;3(2):118-127. doi: $10.2174 / 187221309788489733$ 
3. Omali NB, Subbaraman LN, Coles-Brennan C, et al. Biological and clinical implications of lysozyme deposition on soft contact lenses. Optom Vis Sci. 2015;92(7):750-757. doi:10.1097/ OPX.0000000000000615

4. Lin ST, Mandell RB, Leahy CD, Newell JO. Protein accumulation on disposable extended wear lenses. CLAO J. 1991;17(1):44-50.

5. Luensmann D, Jones L. Albumin adsorption to contact lens materials: a review. Cont Lens Anterior Eye. 2008;31(4):179-187. doi:10.1016/j. clae.2008.05.004

6. Jones L, Evans K, Sariri R, et al. Lipid and protein deposition of $\mathrm{N}$-vinyl pyrrolidone-containing group II and group IV frequent replacement contact lenses. CLAO J. 1997;23(2):122-126.

7. Berry M, Pult H, Purslow C, et al. Mucins and ocular signs in symptomatic and asymptomatic contact lens wear. Optom Vis Sci. 2008;85(10):E930-E938. doi:10.1097/OPX.0b013e318188896b

8. Uno T, Fukuda M, Ohashi Y, et al. Survey of severe contact lens-associated microbial keratitis in Japan. Nippon Ganka Gakkai Zasshi. 2011;115(2):107-115.

9. Sapkota K, Lira M, Martin R, et al. Ocular complications of soft contact lens wearers in a tertiary eye care centre of Nepal. Cont Lens Anterior Eye. 2013;36(3):113-117. doi:10.1016/j. clae.2012.11.002

10. Ozkan J, Rathi VM, de la Jara PL, et al. Effect of daily contact lens cleaning on ocular adverse events during extended wear. Optom Vis Sci. 2015;92(2):157-166. doi:10.1097/OPX.0000000000000479

11. Ueda K, Sahashi N, Takahashi Y, et al. Adherence of cedar pollen and the antigen of cedar pollen to soft contact lenses. J Jpn Cont Lens Soc. 2010;52(2):127-130.

12. Schafer J, Steffen R, Reindel W, et al. Evaluation of surface water characteristics of novel daily disposable contact lens materials, using refractive index shifts after wear. Clin Ophthalmol. 2015;9:1973-1979. doi:10.2147/OPTH.S90376

13. Yue $\mathrm{X}$, Lin $\mathrm{S}, \mathrm{Yu} \mathrm{Y}$, et al. The putative pectin methylesterase gene, BcMF23a, is required for microspore development and pollen tube growth in Brassica campestris. Plant Cell Rep. 2018;37 (7):1003-1009. doi:10.1007/s00299-018-2285-6
14. Mimura T, Sunaga T, Mizota A. Clinical academic topics. Cleaning effect of hydrogen peroxide solution on cedar pollen attached to contact lens. Rinsho Allergy. 2020;40(3):70-79.

15. Vercoulen PHW, Roos RA, Marijnissen JCM, et al. Measuring electric charge on pollen. J Aerosol Sci. 1992;23:377-380. doi:10.1016/ 0021-8502(92)90428-X

16. Bowker GE, Crenshaw HC. Electrostatic forces in wind-pollination Part 2: simulation of pollen capture. Atmos Environ. 2007;41:1596-1603. doi:10.1016/j.atmosenv.2006.10.048

17. Maldonado-Codina C, Efron N. Impact of manufacturing technology and material composition on the surface characteristics of hydrogel contact lenses. Clin Exp Optom. 2005;88(6):396-404. doi:10.1111/ j.1444-0938.2005.tb05106.x

18. Guryca V, Hobzová R, Prádný M, et al. Surface morphology of contact lenses probed with microscopy techniques. Cont Lens Anterior Eye. 2007;30(4):215-222. doi:10.1016/j.clae.2007.02.010

19. Tranoudis I, Efron N. Water properties of soft contact lens materials. Cont Lens Anterior Eye. 2004;27(4):193-208. doi:10.1016/j. clae.2004.08.003

20. Sulley A, Dumbleton K. Silicone hydrogel daily disposable benefits: the evidence. Cont Lens Anterior Eye. 2020;43(3):298-307. doi:10.1016/j.clae.2020.02.001

21. Musgrave CSA, Fang F. Contact lens materials: a materials science perspective. Materials (Basel). 2019;12(2):261. doi:10.3390/ ma12020261

22. Luensmann D, Jones L. Protein deposition on contact lenses: the past, the present, and the future. Cont Lens Anterior Eye. 2012;35(2):5364. doi:10.1016/j.clae.2011.12.005

23. Thekveli S, Qiu Y, Kapoor Y, et al. Structure-property relationship of delefilcon A lenses. Cont Lens Anterior Eye. 2012;35(suppl 1):e14. doi:10.1016/j.clae.2012.08.044

24. Marx S, Lauenborg B, Kern JR. Performance evaluation of delefilcon a water gradient daily disposable contact lenses in first-time contact lens wearers [published online ahead of print, 2018 Feb 6]. Cont Lens Anterior Eye. 2018:S1367-0484(17)30109-1. doi:10.1016/j. clae.2017.12.019
Clinical Optometry

\section{Publish your work in this journal}

Clinical Optometry is an international, peer-reviewed, open access journal publishing original research, basic science, clinical and epidemiological studies, reviews and evaluations on clinical optometry. All aspects of patient care are addressed within the journal as well as the practice of optometry including economic and business analyses. Basic and clinical research papers are published that cover

Submit your manuscript here: https://www.dovepress.com/clinical-optometry-journal all aspects of optics, refraction and its application to the theory and practice of optometry. The manuscript management system is completely online and includes a very quick and fair peer-review system, which is all easy to use. Visit http://www.dovepress.com/ testimonials.php to read real quotes from published authors. 